\title{
Gas Electronegativity Influence on Electrical Breakdown Mechanisms
}

\author{
B. LonČAR ${ }^{a, *}$, R. Radosavluević ${ }^{b}$, M. Vujisić $c^{b}$, K. Stanković ${ }^{b}$ And P. Osmokrović ${ }^{b}$ \\ ${ }^{a}$ Faculty of Technology and Metallurgy, University of Belgrade, Karnegijeva 4, 11120 Belgrade, Serbia \\ ${ }^{b}$ Faculty of Electrical Engineering, University of Belgrade, Bulevar Kralja Aleksandra 73, 11120 Belgrade, Serbia
}

(Received February 2, 2010; in final form December 14, 2010)

\begin{abstract}
This paper discusses the role of different gas breakdown mechanisms depending on electronegativity of the gas, at low values of pressure and inter-electrode gap. Static (dc) electrical breakdown of electropositive, electronegative and noble gases has been investigated theoretically, experimentally and numerically. In the case when the electron mean free path is comparable with characteristic dimension of the electrode system (inter-electrode distance $d$ ), then the breakdown occurs through the Townsend mechanism. In the case when the electron mean free path is much shorter than the characteristic dimension of the electrode system, the breakdown is said to occur through the streamer mechanism. But, between the regions where the breakdown occurs solely by either the Townsend or the streamer mechanism, there is a region where breakdown occurs by the combination of these two mechanisms. The width of this region expressed by the quantity of $p d$ product (product of pressure, $p$, and inter-electrode distance, $d$ ) depends on observed gas tendency to form negative or positive ions (electronegative and noble gases, respectively). The level of the anomalous Paschen effect expression is dependent on whether the observed gas is electronegative or noble. The combined mechanism effects of breakdown and anomalous Paschen effect are quantitatively determined and theoretically explained within this paper.
\end{abstract}

PACS: $51.50 .+\mathrm{v}$

\section{Introduction}

Electrical breakdown of a gas is the result of self-sustained avalanche processes which depend on the relative activity of electron generation and loss mechanisms $[1,2]$. Static (dc) breakdown occurs when the rate of change of the applied voltage is much lower than the rate of elementary processes related to electrical breakdown. The dependence of dc gas breakdown voltage $\left(V_{\mathrm{b}}\right)$ on the product of gas pressure $(p)$ and inter-electrode gap $(d)$ has an asymmetrical $U$ shape and is called the Paschen curve. The minimum of this curve corresponds to the value of the $p d$ product for which net ionization effect is maximal and is referred to as the Paschen minimum [3-8].

If the secondary processes on the electrodes (ionic discharge, photoemission, metastable discharge etc.) dominate the secondary processes in the gas (ionization by positive ions, photoionization, metastable ionization etc.), static breakdown is dominated by the the Townsend mechanism. The condition for the Townsend breakdown to occur is expressed as $[9,10]$ :

* corresponding author; e-mail: bloncar@tmf.bg.ac.rs

$$
\int_{0}^{d} \gamma \exp \left(\int_{0}^{d}(\alpha-\eta) \mathrm{d} x\right) \alpha \mathrm{d} x=1,
$$

where $\alpha$ is the effective coefficient of ionization (number of electronic ionization collisions per unit of length in the direction of the electric field), $\eta$ is the electron attachment coefficient (number of electrons per unit of a length in the direction of the field attached to the electrically negative atoms or molecules), and $\gamma$ is the generalized secondary coefficient of ionization (number of electrons generated from secondary processes per each primary avalanche). These, so-called, avalanche coefficients depend on the electric field profile.

If, however, the secondary processes in the gas dominate, dc breakdown will be in the form of streamers, i.e. determined by the streamer mechanism. The condition for this type of breakdown to occur in a non-homogeneous field is given by the Schumann criterion $[9,10]$ :

$$
\int_{0}^{d}[\alpha(x)-\eta(x)] \mathrm{d} x \geq k
$$

where $k$ is a dimensionless parameter dependent on the gas type [8]. Integrals in expressions (1) and (2) are calculated along the line of the maximum electric field. Especially interesting phenomenon arises in the range on the left of the Paschen minimum, i.e. in its proximity. Within this range the occurrence known as anomalous 
Paschen effect arises. Although this effect is interpreted in the literature [11-14] by complex relation between values of primary and secondary Townsend coefficients, we have developed the model of its interpretation only by the edge breakdowns. Within above mentioned range of $p d$ product, it is possible that spark travels by longer path (of length $l$ ) for which the product $p l$ is equal to product of inter-electrode distance $d$ and pressure $p$ at the Paschen minimum $(p d)_{\text {min }}$. Under such condition, the breakdown will occur at voltage value equal to voltage which corresponds to the Paschen minimum. Since the occurrence of initial electron is of stochastic nature, the breakdown will occur sometimes through the edge lines at described way and sometimes along the line of maximal value of electric field. In the latter case the spark path is shorter (i.e. equal to inter-electrode distance) and the corresponding breakdown voltage will have value higher than voltage at the Paschen minimum. In that sense, statistical sample of random variable "dc breakdown voltage" will follow the mixed distribution of additive type. At even lower values (than those for which anomalous Paschen effect occurs) breakdown becomes of the vacuum type.

\section{The experiment}

The experiment consisted of measuring gas dc breakdown voltage in the proximity of the Paschen minimum, using a gas chamber with an adjustable and replaceable electrode system. The chamber, shown in Fig. 1, consists of a glass tube enclosure with a possibility of adjusting and replacing the electrodes housed in it. After each adjustment of the inter-electrode gap, the chamber would be incorporated into the gas circuit. Before the work pressure adjustment, the chamber was rinsed with working gas, in order to remove any remaining air. The process of rinsing consisted of multistage vacuuming of the chamber (down to $10^{-9}$ bar) and introduction of working gas (up to 1 bar). The chamber designed for underpressure with O-rings treated with vacuum paste and precize manufacturing $(1 \mu \mathrm{m})$ of all parts enabled the stable pressure within the chamber during the one series of measurements. The pressure of He gas, which is the most critical gas from all applied gases because of its very small atom size, was kept constant over $5 \mathrm{~h}$.

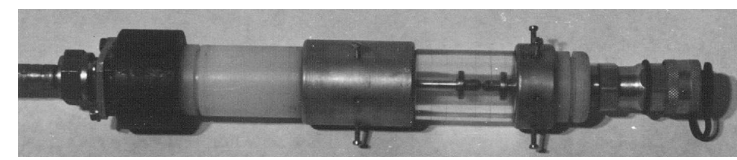

Fig. 1. Chamber with the possibility of adjusting and replacing the electrode system.

Gases used in the experiment were $\operatorname{Ar}(99.995 \%$ purity), $\mathrm{He}\left(99.997 \%\right.$ purity), $\mathrm{N}_{2}\left(99.998 \%\right.$ purity) and $\mathrm{SF}_{6}$ (99.98\% purity), all of which were commercially available. The electrodes were cylindrical with rounded edges, which provided a practically homogeneous electric field in the inter-electrode region. The electrodes were made of electrolytic copper and polished to a high gleam prior to each series of measurements. For determination of the boundary where the electrode effects (work function, topography, surface, melting point, heat conductivity) do not have any further effect on the value of breakdown voltage, i.e. where the Townsend mechanism of breakdown disappears, chambers made of electron, aluminium, silver and tungsten polished to a high gleam or sand blasted were used.

The dc voltage source had a $30 \mathrm{kV}$ maximum voltage, $3 \mathrm{kVA}$ power, and lower than $1 \%$ ripple. The high voltage transformer of the rectifier was powered through a regulation transformer, which enabled adjustment of the dc voltage rise rate, which was $8 \mathrm{kV} / \mathrm{s}$. This value of rise rate enabled gas breakdown which can be treated as dc, since the corresponding time constant of voltage rise is much higher than the time constant of elementary processes in the gas. An electronic control device, with antiparallel thyristors connected in parallel to the primary winding of the transformer, was used for automatic shut-off of the high voltage transformer after breakdown. According to formed budget of measurement uncertainty the obtained results were determined with combined measurement uncertainty less than 5\% [15-17].

The procedure of processing experimental results consisted in: 1 - rejecting of spurious values from all statistical samples, with $5 \%$ level of significance, by applying the Chauvenet criterion, 2 - analyzing adherence of statistical samples to normal, Weibull and exponential distributions by using graphical method, the $\chi^{2}$ test and the Kolmogorov test, 3 - if the test results indicated the possibility of belonging to the mixed type of distribution, additive or multiplicative type, then the samples were separated to subsamples by using the U-test and tested again separately, 4 - obtained results are presented by using histograms and the Paschen curve.

\section{Results and discussion}

In Fig. 2 experimentally obtained points of the Paschen curves are presented for $\mathrm{SF}_{6}$, alongside numerically calculated curves according to the Townsend criterion and Townsend streamer criterion represented by expressions (1) and (2), respectively. Theoretical value of dc breakdown voltage was calculated according to Eqs. (1) and (2) in the following way: (1) Electrical field in the inter-electrode gap was calculated by using the method of charge simulation; (2) Conditions (1) and (2) were verified in the range on the right of the Paschen minimum. According to the Takeshi exponential relations the Townsend coefficients were used for Ar, $\mathrm{He}$ and $\mathrm{N}_{2}$ gases, while the most appropriate linear equation for the observed range of pressure $p$ and inter-electrode distance $d$ was used for $\mathrm{SF}_{6}$ gas [11-13]. Linear increase of potential diference between electrodes was assumed as dependent on the adopted parameter $t$ used during the iterative 
numerical integration. Zeros of obtained functions were determined by the regula falsi method.

Figure 2 shows that experimentally obtained points correspond to the curve calculated numerically according to the streamer criterion down to the point of $8 \mathrm{mbar} \mathrm{mm}$. However to the left of this point, the numerically calculated curve lies above the experimentally obtained points. Therefore, the point 8 mbar $\mathrm{mm}$ can be taken as the boundary for breakdowns which include the pattern of the streamer mechanism. Lower position of the curve calculated according to the Townsend criterion relative to the experimental points may be the result of either the choice of coefficient $\gamma$ or the negligence of electron backscatter effect. Electron backscatter effect originates from scattering of particles in the cathode region that leads to the decrease of the secondary emission coefficient $\gamma$ inversely with respect to electron energy, which, if taken into account, is effectively manifested as an upward shift of the Paschen curve [14]. With the inclusion of this effect, experimental results below $8 \mathrm{mbar} \mathrm{mm}$ adhere to the curve calculated numerically according to the Townsend criterion.

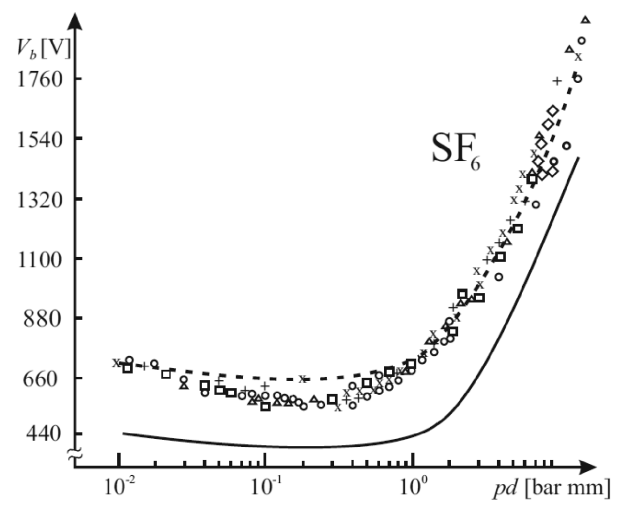

Fig. 2. Experimentally obtained points of the Paschen curve for $\mathrm{SF}_{6}$ gas (dc breakdown, inter-electrode gaps: $\square=0.1 \mathrm{~mm}, \circ=0.2 \mathrm{~mm},+=0.5 \mathrm{~mm}, \mathrm{x}=0.75 \mathrm{~mm}$, $\Delta=1 \mathrm{~mm}$ ) along with the theoretically calculated curves (- Townsend mechanism, - - - streamer mechanism).

The value of 40 mbar mm, which is referred to in literature [14] as the sharp boundary between the Townsend and streamer mechanisms for $\mathrm{SF}_{6}$ gas, does not really represent a clear-cut boundary between these two mechanisms, since neither of them tends to be exclusive around $40 \mathrm{mbar} \mathrm{mm}$. Consequently, a broader range of $p d$ product can be expected to induce breakdowns that would combine the two mechanisms. Such an assumption is supported by the results of the examination of the influence exercised by electrode material and the treatment of electrode surface on the static voltage breakdown value. They clearly indicate that the influence of these parameters disappears above the $p d$ value of 55 mbar mm [9]. Therefore, the Townsend mechanism is relevant for the $\mathrm{SF}_{6}$ breakdown up to the point of 8 mbar mm whereas the combination of the Townsend and streamers mechanisms holds well for the breakdown between the points $8 \mathrm{mbar} \mathrm{mm}$ and $55 \mathrm{mbar} \mathrm{mm}$, although the influence of the Townsend mechanism decreases with the increase of $p d$ value. Above the point of $55 \mathrm{mbar} \mathrm{mm}$, however, the breakdown mechanism becomes exclusively streamers.

Experimentally obtained Paschen curves for $\mathrm{Ar}, \mathrm{He}$ and $\mathrm{N}_{2}$ gases are shown in Figs. 3, 4 and 5 together with corresponding Paschen curves obtained theoretically by using criteria (1) and (2). In the same way as for $\mathrm{SF}_{6}$ gas, the width of transition range where the breakdown occurs by the combination of Townsend and streamer mechanisms was determined. These $p d$ values, representing boundaries between the Townsend and streamer mechanisms, along with the above interpretation, are in agreement with results from other authors [14].

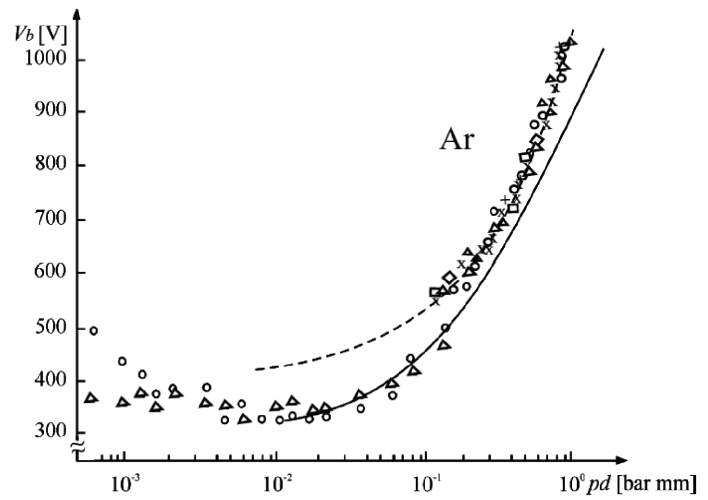

Fig. 3. Experimentally obtained points of the Paschen curve for Ar gas (dc breakdown), for cylindrical and Rogowski shaped electrodes, along with the theoretically calculated curves (- Townsend mechanism, - - streamer mechanism).

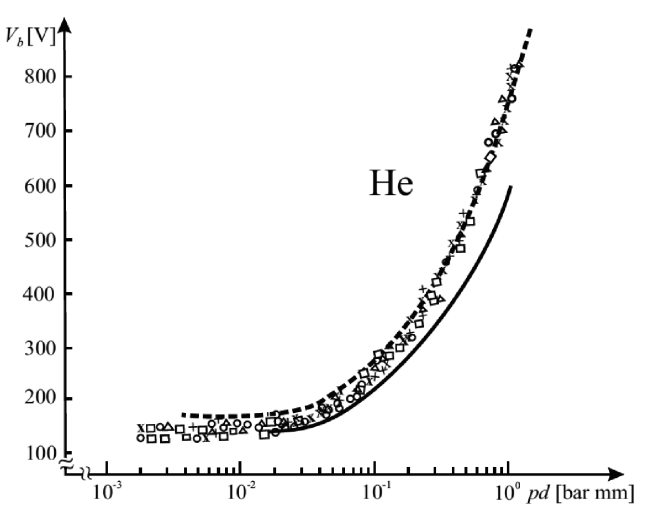

Fig. 4. Experimentally obtained points of the Paschen curves for He (dc breakdown, inter-electrode gaps: $\square=$ $0.1 \mathrm{~mm}, \circ=0.2 \mathrm{~mm},+=0.5 \mathrm{~mm}, \mathrm{x}=0.75 \mathrm{~mm}, \Delta=$ $1 \mathrm{~mm})$, along with the theoretically calculated curves ( - Townsend mechanism, - - - streamer mechanism).

For Ar gas the transition range where the breakdown occurs by the combination of the Townsend and 


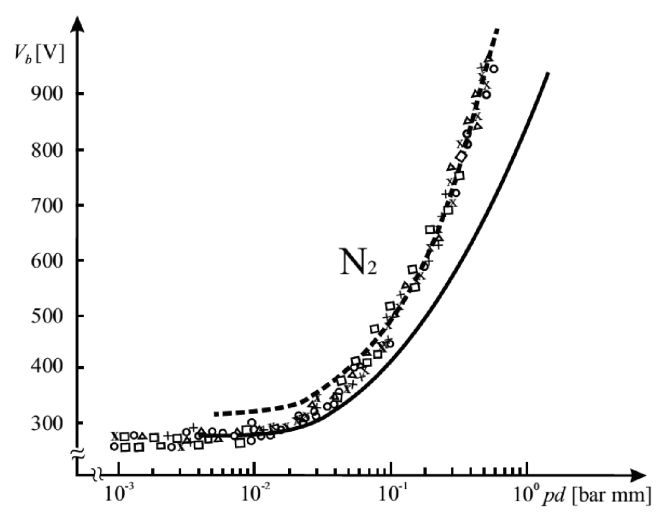

Fig. 5. Experimentally obtained points of the Paschen curves for $\mathrm{N}_{2}$ (dc breakdown, inter-electrode gaps: $\square=$ $0.1 \mathrm{~mm}, \circ=0.2 \mathrm{~mm},+=0.5 \mathrm{~mm}, \mathrm{x}=0.75 \mathrm{~mm}, \Delta=$ $1 \mathrm{~mm})$, along with the theoretically calculated curves ( - Townsend mechanism, - - - streamer mechanism).

streamer mechanisms lies between 200 mbar mm and $400 \mathrm{mbar} \mathrm{mm}$, while for He gas this range is between $100 \mathrm{mbar} \mathrm{mm}$ and $200 \mathrm{mbar} \mathrm{mm}$, and for $\mathrm{N}_{2}$ gas between $10 \mathrm{mbar} \mathrm{mm}$ and $50 \mathrm{mbar} \mathrm{mm}$. The level of anomalous discharges, which we have interpreted by the edge effects, is the most expressed in the case of He and Ar gases, and minimally expressed in the case of $\mathrm{SF}_{6}$.

Experimentally obtained results of static breakdown voltages in the proximity of the Paschen minimum to the left of it are presented in Fig. 6a for $\mathrm{He}(d=2.4 \mathrm{~mm}$, $p=1.12$ mbar $)$ and in Fig. 7a for $\mathrm{N}_{2}(d=1 \mathrm{~mm}$, $p=2$ mbar). The corresponding results obtained by computer simulation according to our model are shown in Figs. $6 \mathrm{~b}$ and $7 \mathrm{~b}$, respectively for $\mathrm{He}$ and $\mathrm{N}_{2}$. Figures 6 and 7 demonstrate that in the area left of the Paschen minimum the "breakdown voltage" random variable follows a cumulative distribution of the additive type. This conclusion is substantiated by the results shown in Fig. 8, in which breakdown voltages in the proximity of the Paschen minimum to the left of it, for one series of measurements with $\mathrm{SF}_{6}$ gas, are presented against the Weibull probability scale $(d=1 \mathrm{~mm}, p=0.1 \mathrm{mbar})$. It can clearly be seen from this figure that the "breakdown voltage" random variable belongs to the mixed Weibull distribution of the additive type. It is obvious that on the right of the Paschen minimum the same results were obtained for both types of electrodes.

\section{Conclusion}

Results obtained showed that in the range on the right of the Paschen minimum electrical breakdown occurs either through streamer mechanism (at higher values of $p d$ product) or through the Townsend mechanism (at lower values of $p d$ product) independently of the applied gas. Transition from streamer to the Townsend mechanism appears at first when electronegative gas is applied and at last when noble gas is applied. This result is easy to

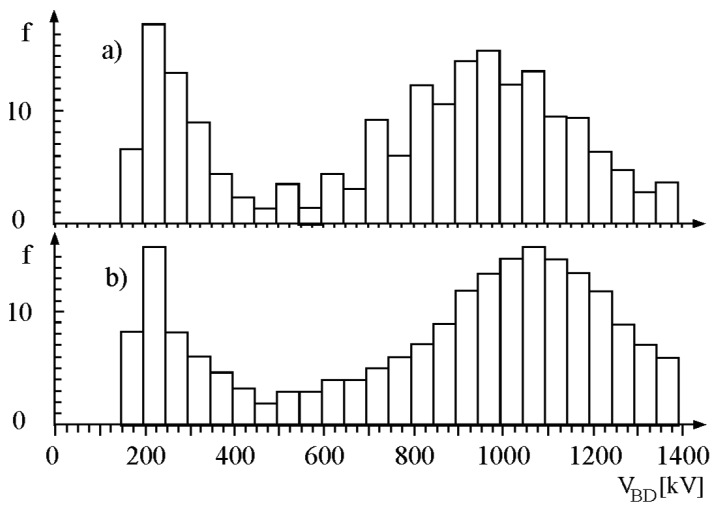

Fig. 6. Histogram of dc breakdown voltages at the points left of the Paschen minimum for He gas $(d=$ $2.4 \mathrm{~mm}, p=11.2 \mathrm{mbar}$ ): (a) experimentally obtained results, (b) numerically calculated results.

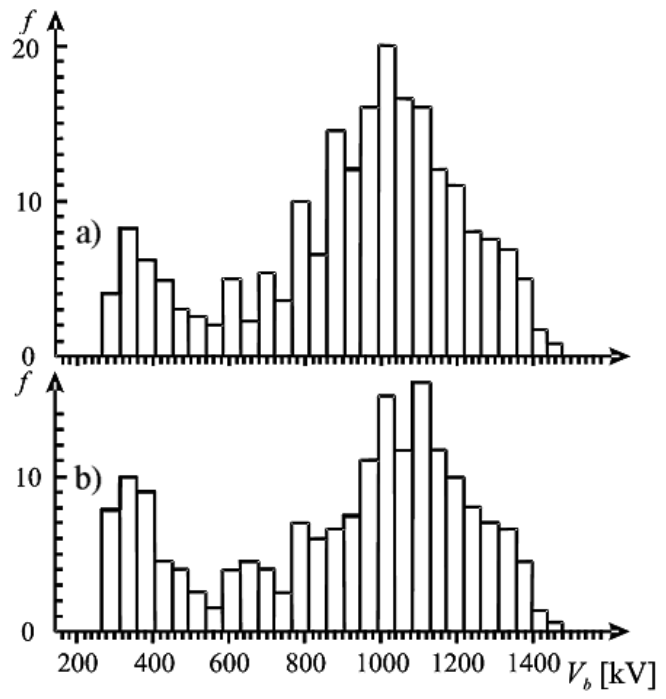

Fig. 7. Histogram of dc breakdown voltages at the points left of the Paschen minimum for $\mathrm{N}_{2}$ gas $(d=$ $1 \mathrm{~mm}, p=2 \mathrm{mbar}$ ): (a) experimentally obtained results, (b) numerically calculated results.

explain by the synergistic effects of large number of free electrons needed for the formation of streamer forehead in the case of noble gases, their small number in the case of electronegative gases and the expressed sensitivity of electronegative gases on the occurrence of cold emission. Also, it is shown that for all applied gases there exists one transition range where the breakdown occurs by the combination of these two mechanisms. This transition range is the widest for electronegative gases and the narrowest for noble gases.

In the range on the left of the Paschen minimum the breakdown occurs through the Townsend mechanism, but phenomenon known as anomalous Paschen effect appears. According to our interpretation, anomalous Paschen effect is occurrence at which the spark travels by 


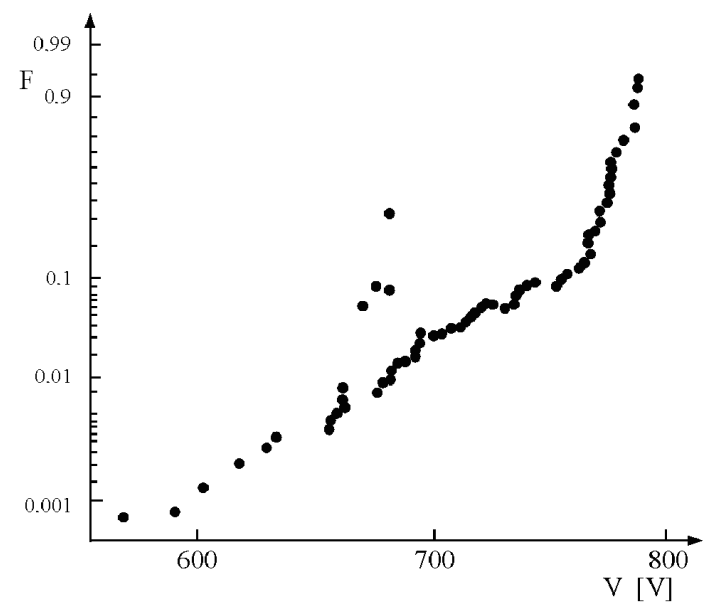

Fig. 8. Experimentally obtained results for dc breakdown voltages of one series of measurements presented on the Weibull probability scale $(d=1 \mathrm{~mm}, p=$ 0.1 mbar, $\mathrm{SF}_{6}$ gas).

longer path which is energetically favourable, being the case of physical phenomena. Occurrence of anomalous Paschen effect, i.e. edge effects, is the most expressed for noble gases, while for electronegative gases is minimally expresed. The fact that this effect is the most expressed for noble gases is the consequence of their small value of ionisation potential which implies the existence of large number of free, potentially initiatory electrons. These electrons make possible the occurrence of breakdown always along the most favourable energetic path, i.e. along the edge lines of the electrical field, whose length multiplied with pressure value corresponds to the position of minimum. That is not the case for electronegative gases, when the occurrence of breakdown appears in the central electrode regions. The reason is the volume larger than that corresponding to the edge lines of electrical field, which is the consequence of deficit in free electrons for this sort of gases.

\section{Acknowledgments}

The Ministry for Science and Technological Development of the Republic of Serbia supported this work under contract 171007 .

\section{References}

[1] J.M. Meek, J.D. Crags, Electrical Breakdown of Gases, Wiley, New York 1978.

[2] A. Von Engel, Ionized Gases, Clarendon Press, Oxford 1965.

[3] B. Loncar, P. Osmokrovic, A. Vasic, S. Stankovic, IEEE Trans. Plasma Sci. 34, 1561 (2006).

[4] P. Osmokrovic, A. Vasic, IEEE Trans. Plasma Sci. 33, 1672 (2005).

[5] M.M. Pejovic, C.S. Milosavljevic, IEEE Trans. Plasma Sci. 31, 776 (2003).

[6] W.O. Schumann, Elektrische Durchbruchfel-stärke von Gasen, Springer-Verlag, Berlin 1923.

[7] M.J. Schonhuber, IEEE Trans. Pow. Apparat. Syst. 88, 100 (1969).

[8] P. Osmokrovic, IEEE Trans. Plasma Sci. 21, 645 (1993).

[9] P. Osmokrovic, IEEE Trans. Pow. Deliv. 4, 2095 (1989).

[10] P. Osmokrovic, B. Loncar, R. Sasic, IEEE Trans. Plasma Sci. 33, 1729 (2005).

[11] A. Pedersen, IEEE Trans. Electr. Insul. 24, 721 (1989).

[12] P. Osmokrovic, N. Kartalovic, IEEE Trans. Power Syst. 12, 1455 (1997).

[13] P. Osmokrovic, I. Krivokapic, S. Krstic, IEEE Trans. Dielectr. Electr. Insul. 1, 77 (1994).

[14] K.F. Geibig, Ph.D. Thesis, T.U. Karlsruhe, 1982.

[15] K. Stankovic, M. Vujisic, Nucl. Technol. Radiat. 23, 41 (2008).

[16] K. Stankovic, M. Vujisic, E. Dolicanin, Nucl. Technol. Radiat. 24, 132 (2009).

[17] K. Stankovic, M. Vujisic, Lj. Delic, Nucl. Technol. Radiat. 25, 46 (2010). 